\title{
Epidemiology, features and outcome of pain in patients with advanced hematological malignancies followed in a home care program: an Italian survey
}

\author{
Pasquale Niscola • Claudio Cartoni • Claudio Romani • \\ Gregorio Antonio Brunetti • Gianna Maria D'Elia • \\ Luca Cupelli • Andrea Tendas • Paolo de Fabritiis • \\ Franco Mandelli • Robin Foà
}

Received: 3 February 2007 / Accepted: 3 April 2007 / Published online: 21 April 2007

(C) Springer-Verlag 2007

\begin{abstract}
We report on epidemiology, features, outcome, and domiciliary management of pain in patients with advanced hematological malignancies followed by an experienced hospital-based home care (HC) team. Out of 469 patients, $244(52 \%)$ experienced a total of 284 pain syndromes. Pain intensity was rated from mild to moderate in $31 \%$ and from moderate to severe in $69 \%$ of them. The diagnosed pain mechanisms were deep somatic in $56 \%$, superficial somatic in $15 \%$, visceral $14 \%$, mixed $8 \%$, and neuropathic in $7 \%$ of pain syndromes, respectively. Incident pain was observed in $38 \%$ of all pain syndromes. In every diagnostic group, deep somatic pain was prevalent. Moreover, $85 \%$ of visceral pain syndromes were observed in patients affected by non-Hodgkin's lymphoma (NHL). In addition, out of 284 pain syndromes, 150 (51\%) were caused by bone involvement. The most frequent recognized pain provocative mechanisms were bone marrow expansions, osteolysis, lymph node enlargement, and mucositis. In our experience, an approach based on the association of
\end{abstract}

P. Niscola $(\bowtie) \cdot$ L. Cupelli $\cdot$ A. Tendas $\cdot$ P. de Fabritiis

Haematology Division, Tor Vergata University,

Sant'Eugenio Hospital,

Rome, Italy

e-mail: pasquale.niscola@uniroma2.it

C. Cartoni · G. A. Brunetti · G. M. D'Elia · F. Mandelli • R. Foà

Human Biopathology and Haematology Department,

"La Sapienza" University,

Rome, Italy

C. Romani

Armando Businco Cancer Centre,

Cagliari, Italy causal therapies and analgesics allows optimal control of most pain syndromes. Therefore, pain is a major problem in patients affected by advanced hematological malignancies, and its management can be effective and feasible when carried out by a skilled HC team.

Keywords Home care $\cdot$ Pain $\cdot$ Hematological malignancies

\section{Introduction}

So far, pain epidemiology in patients with advanced hematological malignancies, other than multiple myeloma (MM), has been poorly investigated (Table 1). This symptom has traditionally been believed to be a rare manifestation compared to solid tumor settings [1-3]. Nevertheless, more recent studies have shown a high prevalence of this symptom in the hematological population [4-7], in which the occurrence of specific pain syndromes has been described [8]. However, no study on this topic concerning patients with blood-related neoplasm in the home care (HC) setting has been reported. To address this issue, we conducted a 6-year prospective study on the domiciliary management of a cohort of patients with advanced bloodrelated neoplasm followed at home by a hospital-based multidisciplinary $\mathrm{HC}$ team $[9,10]$.

\section{Materials and methods}

The study included 469 patients followed at home over a 6-year period by a team composed of seven hematologists, ten nurses, two psychologists, one social worker, and 
Table 1 Pathophysiology of the most common pain syndromes in patients with hematological malignancies

\begin{tabular}{|c|c|c|}
\hline \multicolumn{2}{|l|}{ Pain type } & \multirow{2}{*}{$\begin{array}{l}\text { Pain origin and syndromes } \\
\text { Bone marrow expansion and osteolysis. Spleen and liver capsulae distension by tumor infiltration and } \\
\text { organ enlargement. Headache by liquor loss (post dural puncture); intracranial hypertension } \\
\text { (meningeal and/or brain tumor involvement) }\end{array}$} \\
\hline Nociceptive & Deep somatic & \\
\hline & Superficial somatic & Mucositis, cutaneous lesions \\
\hline & Visceral & Infiltration and/or compression of viscera cava by abdominal nodes, spleen, and liver enlargement \\
\hline \multirow[t]{2}{*}{ Neuropathic } & Peripheral neuropathic & $\begin{array}{l}\text { Vinca alkaloids, thalidomide and bortezomib toxicity. Post-herpetic neuralgia. Neuropathies due to } \\
\text { para-proteins. Amyloidosys. Plexopathy by tumor invasion and/or node enlargement compression } \\
\text { (lymphomas) }\end{array}$ \\
\hline & Central Neuropathic & CNS damage and/or tumor involvement \\
\hline Mixed & Neuropathic + somatic & Meningosis, peripheral nerve damage, and/or tumor involvement \\
\hline \multirow[t]{2}{*}{ Breakthrough } & $\begin{array}{l}\text { Incident (related to the } \\
\text { movement) }\end{array}$ & Osteolysis, mucositis, cutaneous derangement \\
\hline & Non incident pain & Transient pain flares during stable analgesia \\
\hline
\end{tabular}

several other care providers trained in palliative care. A physician and a nurse were available on duty $24 \mathrm{~h}$ a day all year round. The HC process was based on specific internal operating models and team guidelines to ensure continuous efforts toward the best quality of care [10]. The decision to follow a patient at home was taken together by the referral hematologist and $\mathrm{HC}$ team. The availability at home of a reliable and educated caregiver has been considered an essential condition to activate a $\mathrm{HC}$ program. Regular weekly team meetings allowed the examination of emerging problems and to refine treatment and care objectives. The $\mathrm{HC}$ team worked together with the general services and diagnostic structures of the Human Biopathology and Haematology Department, "La Sapienza" University, Rome. In addition, a close collaboration with a hospice guaranteed periodical family relief and the palliation of specific refractory symptoms requiring measures unavailable at home.
From the assessment up to the end-life care, precise decisions have been implemented and applied [10], in particular regarding pain therapy, transfusion policy, infection treatments, and bleeding management in all phases of $\mathrm{HC}$ processes.

Pain, as the fifth vital sign, was assessed, during a clinical visit, every $24 \mathrm{~h}$ until analgesia and then every 3 days. The pain intensity was reported by a Numerical Analogue Scale (NRS), which rated 0 (no pain), 1 to 3 (mild pain), 4 to 6 (moderate), and 7 to 10 (severe). In less reliable patients, only a verbal description scale including four items: "no pain," "mild pain," "moderate," and "severe pain" [11] was applied. In nonverbal patients, like young children and some cognitive-impaired elderly, a face pain scale [12] and, in very few cases, the parent ratings (four items as above) as proxy measures [13] were adopted. Each pain syndrome was properly assessed and classified by the treating physicians as deep somatic, superficial somatic,

Table 2 Patient's demographic features, incidence, and distribution of pain syndromes according to the diagnosis

\begin{tabular}{|c|c|c|c|c|c|c|}
\hline \multirow[t]{2}{*}{ Malignancy } & \multicolumn{2}{|c|}{ Patients } & \multirow{2}{*}{$\begin{array}{l}\mathrm{M} / \mathrm{F} \\
\text { No. }\end{array}$} & \multirow{2}{*}{$\begin{array}{l}\text { Mean Age (SD) } \\
\text { Years }\end{array}$} & \multirow{2}{*}{$\begin{array}{l}\text { Incidence of pain } \\
\text { Percent }\end{array}$} & \multirow{2}{*}{$\begin{array}{l}\text { Pain syndromes } \\
\text { No. }\end{array}$} \\
\hline & No. & Percent & & & & \\
\hline NHL & 128 & 27 & $71 / 57$ & 61 (17) & 60 & 89 \\
\hline AML & 69 & 15 & $38 / 31$ & $63(20)$ & 41 & 34 \\
\hline MDS & 63 & 13 & $32 / 31$ & $78(7)$ & 32 & 22 \\
\hline $\mathrm{BC}$ & 56 & 12 & $30 / 26$ & $61(15)$ & 52 & 34 \\
\hline ALL & 39 & 8 & $23 / 16$ & $29(21)$ & 79 & 40 \\
\hline MM & 39 & 8 & $19 / 20$ & $71(9)$ & 90 & 38 \\
\hline CMPD & 33 & 7 & $22 / 11$ & $78(9)$ & 18 & 8 \\
\hline CLL & 24 & 5 & $13 / 11$ & $73(8)$ & 42 & 11 \\
\hline HD & 11 & 3 & $6 / 5$ & $50(27)$ & 45 & 5 \\
\hline Others & 7 & 2 & $4 / 3$ & $66(16)$ & 43 & 3 \\
\hline Total & 469 & 100 & $258 / 211$ & $63(20)$ & 52 & 284 \\
\hline
\end{tabular}

$M M$ Multiple myeloma, $A L L$ acute lymphoblastic leukemia, $N H L$ non-Hodgkin's lymphomas; $B C$ blastic crisis, $H D$ Hodgkin's disease, $C L L$ chronic lymphocytic leukemia, acute myeloblastic leukemia, $M D S$ myelodysplastic syndromes, $C M P D$ chronic myeloproliferative disorders 
Table 3 Neurophysiological classification of pain syndromes and their distribution according to the diagnosis

\begin{tabular}{|c|c|c|c|c|c|c|c|c|c|c|c|}
\hline \multirow[t]{3}{*}{ Pain } & \multicolumn{11}{|c|}{ Malignancy } \\
\hline & \multicolumn{11}{|l|}{ No. $(\%)$} \\
\hline & $\mathrm{MM}$ & ALL & NHL & $\mathrm{BC}$ & HD & CLL & AML & CMPD & MDS & Oth & Total \\
\hline DS & $30(80)$ & $20(50)$ & $35(39)$ & $20(59)$ & $5(100)$ & $18(57)$ & $10(77)$ & $12(60)$ & $6(60)$ & $3(100)$ & $159(56)$ \\
\hline SS & $3(8)$ & $8(20)$ & $10(11)$ & $6(17)$ & 0 & $7(22)$ & $2(15)$ & $5(25)$ & $1(10)$ & 0 & $42(15)$ \\
\hline Vis & $1(2)$ & 0 & $33(37)$ & 0 & 0 & $1(3)$ & $1(8)$ & $1(5)$ & $2(20)$ & 0 & 39 (14) \\
\hline NP & $1(2)$ & $5(12)$ & $4(5)$ & $4(12)$ & 0 & $4(12)$ & 0 & $1(5)$ & $1(10)$ & 0 & $20(7)$ \\
\hline Mix & $3(8)$ & $7(18)$ & $7(8)$ & $4(12)$ & 0 & $2(6)$ & 0 & $1(5)$ & 0 & 0 & $24(8)$ \\
\hline Total & $38(100)$ & $40(100)$ & 89 (100) & $34(100)$ & $5(100)$ & $32(100)$ & $13(100)$ & $20(100)$ & $10(100)$ & $3(100)$ & $284(100)$ \\
\hline
\end{tabular}

$D S$ Deep somatic, SS superficial somatic, Vis visceral, NP neuropathic pain, Mix mixed

visceral, neuropathic, and mixed or unknown [14, 15]. Incidental pain development, which was related to movements, was also assessed.

A treatment protocol based on the World Health Organization analgesic ladder [16] and according to the principles of opioid management for onco-hematological patients [17] was applied in association with causal measures if required. Patients whose pain was considered mild were treated with acetaminophen (if thrombocytopenic) or nonsteroidal anti-inflammatory drugs; for moderate pain, weak opioids were used; strong opioids were administered to manage severe pain. Adjuvant drugs were added according to the neuropathic features of pain for each step. No devices were implanted.

Pain control was defined by almost $50 \%$ reduction in pain intensity compared to baseline and to a NRS $<5$. The data were collected, and a computer-based statistical analysis was performed. The parametric and nonparametric variables were analyzed by the Student's $t$ test and the chisquare test, respectively. $p$ values $<0.05$ were considered statistically significant.

\section{Results}

Diagnoses are shown in Table 2. There were 258 (55\%) men, with a median age of 67 (4-95) years; the median Karnofsky performance status (KPS) was 50 (10-70). Patients were properly informed about the aims of the study and gave their consent.

They were followed at home for a mean of $72 \pm 144$ (range 1-1,132) days; 368 (85\%) patients died at home and $65(15 \%)$ after a hospital admission. Out of 469 patients, $244(52 \%)$ experienced almost one pain syndrome, for a total of 284 , the intensity of which was rated from mild to moderate in $92(31 \%)$ and from moderate to severe in $192(69 \%)$.

Patients who experienced pain were significantly younger than those without pain $(57 \pm 21$ vs $69 \pm 16, p<0.0005)$. Moreover, a significantly higher incidence of pain was recorded, in 49 patients younger than 20, compared to others $(85 \%$ vs $49 \%, p=0.0007)$.

Patients affected by MM, acute lymphoblastic leukemia (ALL), NHL, and blastic crisis (BC) had a higher incidence of pain compared to other disease groups $(p=0.0011)$.
Table 4 Pathogenetic mechanisms and their distribution according to neurophysiological classification

$D S$ Deep somatic; SS superficial somatic; Visc visceral; NP neuropathic; $B M$ bone marrow; $Z P$ zoster pain; $P H N$ postherpetic neuralgia

\begin{tabular}{lllllll}
\hline Cause & \multicolumn{7}{l}{ Kind of pain } & & & \\
& \multicolumn{1}{l}{ DS } & SS & Visc & NP & Mixed & Total \\
& No. (\%) & & & & & \\
\hline BM expansion & $80(50)$ & 0 & 0 & 0 & $13(54)$ & $93(33)$ \\
Osteolysis & $44(27)$ & 0 & 0 & 0 & $2(9)$ & $46(16)$ \\
Lymphoadenomegaly & 0 & 0 & $33(85)$ & 0 & $3(12)$ & $36(13)$ \\
Mucositis & 0 & $31(74)$ & 0 & 0 & 0 & $31(11)$ \\
ZP and PHN & 0 & 0 & 0 & $18(90)$ & 0 & $18(6)$ \\
Organ enlargements & $14(9)$ & 0 & 0 & 0 & 0 & $14(5)$ \\
Meningosis & $8(5)$ & 0 & 0 & 0 & $5(21)$ & $13(5)$ \\
Cutaneous ulcers & 0 & $11(26)$ & 0 & 0 & 0 & $11(4)$ \\
Infections & $7(5)$ & 0 & 0 & 0 & 0 & $7(2)$ \\
Neuropathies & 0 & 0 & 0 & $2(10)$ & 0 & $2(0.7)$ \\
Others & $6(4)$ & 0 & $6(15)$ & 0 & $1(4)$ & $12(4.3)$ \\
Total & $159(100)$ & $42(100)$ & $39(100)$ & $20(100)$ & $24(100)$ & $284(100)$ \\
& & & & & &
\end{tabular}


Table 5 Pathogenetic mechanisms of pain

$C N$ Continuous nociceptive; $M I$ mixed incident; $P I$ pure incident; $N P$ neuropathic

\begin{tabular}{lllllll}
\hline Causes & Pain & & & & & \\
\cline { 2 - 6 } & CN & MI & PI & NP & Mixed & Total \\
& No. (\%) & & & & & \\
\hline BM expansion & $70(52)$ & $10(10)$ & 0 & 0 & $13(54)$ & $93(33)$ \\
Osteolysis & 0 & $41(41)$ & $3(60)$ & 0 & $2(9)$ & $46(16)$ \\
Lymphoadenomegaly & $33(25)$ & 0 & 0 & 0 & $3(12)$ & $36(13)$ \\
Mucositis & 0 & $31(31)$ & 0 & 0 & 0 & $31(11)$ \\
ZP and PHN & 0 & 0 & 0 & $18(90)$ & 0 & $18(6)$ \\
Organ enlargements & $14(10)$ & 0 & 0 & 0 & 0 & $14(5)$ \\
Meningosis & $8(6)$ & 0 & 0 & 0 & $5(21)$ & $13(5)$ \\
Cutaneous ulcers & 0 & $11(10)$ & 0 & 0 & 0 & $11(4)$ \\
Infections & $3(2)$ & $4(4)$ & 0 & 0 & 0 & $7(2)$ \\
Neuropathies & 0 & 0 & 0 & $2(10)$ & 0 & $2(0.7)$ \\
Others & $6(5)$ & $4(4)$ & $2(40)$ & 0 & $1(4)$ & $5(4.3)$ \\
Total & $134(100)$ & $101(100)$ & $5(100)$ & $20(100)$ & $24(100)$ & $284(100)$ \\
& & & & & & \\
\hline
\end{tabular}

Among patients with acute leukemias, ALL patients had a higher incidence of pain compared to myeloblastic subtypes ( $79 \%$ vs $41 \%, p=0.0002$; Table 2 ).

Pain syndromes were diagnosed as deep somatic (56\%), superficial somatic (15\%), visceral (14\%), neuropathic (7\%), and mixed (8\%). In all diagnosis groups, a higher incidence of deep somatic pain has been recorded (Table 3). Moreover, out of 39 visceral pain states, $33(85 \%)$ have been observed in NHL patients and $6(15 \%)$ in others $(p=0.0001)$.

The occurrence of incidental pain, including pure and mixed incidental (continuous nociceptive plus incidental pain), was recorded in 106/284 (38\%) of pain syndromes with a higher rate in MM patients, in which this pain feature was recorded in $32(85 \%)$ out of 38 of pain syndromes. The causative mechanisms of pain were directly referable to underlying diseases, to their clinical complications, or other unrelated conditions in 69,22 , and $9 \%$ of pain syndromes, respectively (Tables 4 and 5 ). The most involved sites and the percentages of the respectively afflicting pain syndromes (Table 6) were as follows: spine $(27 \%)$, abdomen $(20 \%)$, legs $(15 \%)$, thorax $(11 \%)$, orofaringeal tract $(10 \%)$, diffuse $(7 \%)$, and others $(10 \%)$.

Table 6 Sites of pain

\begin{tabular}{lrr}
\hline Site & No. & $\%$ \\
\hline Spine & 76 & 27 \\
Abdomen & 56 & 20 \\
Lower extremities & 43 & 15 \\
Thorax & 31 & 11 \\
Orofaringeal cable & 31 & 10 \\
Diffuse & 20 & 7 \\
Head and facial bone & 20 & 7 \\
Pelvis bones & 5 & 2 \\
Cervical region & 2 & 1 \\
Total & 284 & 100 \\
\hline
\end{tabular}

No significant differences were found in the length of HC stay results between patients suffering from pain compared to others (67 vs 76 days, $p=0.46$ ).

The pain management was entirely provided at home, given that no truly intractable pain requiring hospital admission was observed. Although an effective pain control was attained in 259/284 (92\%) of pain syndromes, a completely stable pain relief was achieved in 202/284 $(71 \%)$ of them. A lower response rate was found in the presence of neuropathic and incidental features (Table 7). Indeed, in the group of patients presenting these poor prognostic features compared to those with only continuous nociceptive pain, a completely stable pain relief was attained in $69 / 150(46 \%)$ vs $132 / 135(98 \% ; p=0.0001)$.

\section{Discussion}

Our data indicate that pain is a major concern for patients suffering from advanced hematological malignancies; therefore, its management should be one of the most important aspects of a domiciliary palliative care program [18]. Moreover, according to the type of underlying malignancy and to the pattern of the disease involvement, different painful syndromes, sometimes overlapping, may be distinguished [7]. Two main pain types can be dis-

Table 7 Response to treatment according the typology of pain

\begin{tabular}{lllll}
\hline Pain control & $\begin{array}{l}\text { Continuous } \\
\text { nociceptive }\end{array}$ & Incident & $\begin{array}{l}\text { Neuropathic } \\
\text { mixed }\end{array}$ & Total \\
\hline $\begin{array}{c}\text { Sufficient to } \\
\text { good (\%) }\end{array}$ & $132(98)$ & $42(40)$ & $27(61)$ & $202(71)$ \\
$\begin{array}{l}\text { Poor (\%) } \\
\text { Total (\%) }\end{array}$ & $2(2)$ & $64(60)$ & $17(29)$ & $82(29)$ \\
& $134(100)$ & $106(100)$ & $44(100)$ & $284(100)$ \\
\hline
\end{tabular}


tinguished, based on the causative mechanisms: nociceptive (somatic and visceral) pain, mediated by the activation of normal nerve endings, and neuropathic pain caused by injuries to the peripheral or central nervous system. The main issue in differentiating neuropathic from nociceptive pain is to determine appropriate pharmacological management, given that most neuropathic pains may require high doses of opioids and the addition of an adjuvant analgesic regime (antidepressant or anticonvulsant drugs) [17].

The occurrence of incidental pain, which is a severe movement-induced transient pain flare, should be evaluated in the assessment of each pain syndrome, although the patient may be relatively comfortable at rest. This kind of pain, in our experience, afflicted most of the patients with skeletal damages and those presenting mucositis and skin ulcers. It has been described as a heterogeneous phenomenon, which may be a marker for more severe pain syndromes, being associated with functional impairment, psychological distress, and poor pain control [7, 14, 19].

Regarding the provocative mechanisms, skeletal lesions afflicted most MM patients, who suffered from localized and sometimes irradiated nociceptive continuous pain at rest, complicated by neuropathic symptoms (mixed pain) and incidental pain.

Node enlargement, visceral localizations, and spleen and liver capsulae distension are typically recorded in NHL patients and, to a minor extent, in those affected by other chronic lympho-proliferative disorders and chronic myeloproliferative malignancies. The meningeal and/or brain localization, mainly recorded in ALL patients, causes, in most cases, diffuse headache due to stretching and compression of the meninges, intracranial veins, and meningeal arteries (deep somatic pain), or to the tumor growth around cranial nerves (neuropathic pain). In our series, the overall incidence of pain was $52 \%$, with higher values recorded in patients affected by MM, ALL, NHL, and BC. Among 258 patients experiencing pain, $35(14 \%)$ presented more than one pain syndrome, underlining that pain states related to hematological malignancies may be due to very heterogeneous and sometimes coexisting mechanisms. Skeletal damage and/or bone marrow (BM) involvement are characteristics of these diseases, and as expected, the incidence of deep somatic pain was higher among patients with MM, ALL, BC, and acute myeloid leukemia (AML). However, the sites of pain were uniformly distributed; spine and abdomen were the most afflicted anatomical regions.

Even in the hematological setting, pain can become a compelling clinical problem. Indeed, we found that 150/284 $(53 \%)$ of all diagnosed pain syndromes were complicated by poor prognostic factors, such as neuropathic and incidental pain features, associated with poor pain control by opioids [20]. Moreover the pain intensity was rated as 5 or above (moderate to severe pain) in $69 \%$, and those values are considered substantial and interfering with the quality of life [11]. Although the study has not been designed to address the treatment of pain in $\mathrm{HC}$, we found that a comprehensive approach based on a multidisciplinary and structured HC service and on standardized protocols was feasible and allowed for acceptable pain relief at home in more than $90 \%$ of pain syndromes, included those having poor prognosis. In conclusion, in the setting of advanced hematological patients referred to a HC program, pain represents a challenging concern for which domiciliary management can be a feasible and effective solution when carried out by a skilled HC team.

Acknowledgements We are indebted with Ms. Carla Fritz for the editorial assistance and for the language revision of the manuscript.

\section{References}

1. Portenoy RK (1989) Cancer pain: epidemiology and syndromes. Cancer 63:2298-2307

2. Bonica JJ (1990) Cancer pain. In: Bonica JJ (ed) The management of pain. Lea and Febiger, Malvern PA, pp 400-460

3. Twycross R, Harcourt J, Bergl S (1996) A survey of pain in patients with advanced cancer. J Pain Symptom Manage 12:273-282

4. Vainio A, Auvinen A (1996) Prevalence of symptoms among patients with advanced cancer: an international collaborative study. Symptom Prevalence Group. J Pain Symptom Manage 12(1):3-10 (Jul)

5. Bauduer F, Capdupuy C, Renoux M (2000) Characteristics of deaths in a department of oncohaematology within a general hospital. A study of 81 cases. Support Care Cancer 8(4):302-306 (Jul)

6. Stalfelt AM, Brodin H, Pettersson S, Eklof A (2003) The final phase in acute myeloid leukaemia (AML). A study on bleeding, infection and pain. Leuk Res 27(6):481-488 (Jun)

7. Niscola P, Romani C, Cartoni C, Cupelli L, Piccioni D, Dentamaro T, Scaramucci L, Tendas A, Tolu B, Giovannini M, Perrotti AP, de Fabritiis P (2007) Epidemiology of pain in the hospital haematological setting: an Italian survey. Leuk Res DOI 10.1016/j.leukres.2007.03.024

8. Niscola P, Arcuri E, Giovannini M, Scaramucci L, Romani C, Palombi F et al (2004) Pain syndromes in haematological malignancies: an overview. Hematol J 5(4):293-303

9. Cartoni C, Brunetti GA, D'Elia GM et al (2002) Home care for patients with hematologic malignancies. (Abstract 254). Abstract book of the seventh annual meeting of the European Haematology Association, Florence (Italy). The Haematology Journal 3(Suppl 1):84

10. Niscola P, de Fabritiis P, Cartoni C, Romani C, Sorrentino F, Dentamaro T, Piccioni D, Scaramucci L, Giovannini M, Amadori S, Mandelli F (2006) Home care management of patients affected by haematological malignancies: a review. Haematologica/The Hematology Journal 91(11):1525-1531

11. Caraceni A, Cherny N, Fainsinger R et al (2002) Pain measurement tools and methods in clinical research in palliative care: recommendations of an Expert Working Group of the European Association of Palliative Care. J Pain Symptom Manage 23(3):239-255 (Mar)

12. Kim EJ, Buschmann MT (2006) Reliability and validity of the Faces Pain Scale with older adults. Int J Nurs Stud 43(4):447-456 (May)

13. Voepel-Lewis T, Malviya S, Tait AR (2005) Validity of parent ratings as proxy measures of pain in children with cognitive impairment. Pain Manag Nurs 6(4):168-174 (Dec) 
14. Caraceni A, Weinstein SM (2001) Classification of cancer pain syndromes. Oncology 15(12):1627-1640, 1642 (Dec)

15. Caraceni A, Portenoy RK (1999) An international survey of cancer pain characteristics and syndromes. IASP Task Force on Cancer Pain. International Association for the Study of Pain. Pain 82(3):263-274 (Sep)

16. World Health Organization (1986) Cancer pain relief. World Health Organization, Geneva

17. Niscola P, Scaramucci L, Romani C, Giovannini M, Maurillo L, Del Poeta G, Cartoni C, Arcuri E, Amadori S, De Fabritiis P
(2006) Opioids in pain management of blood-related malignancies. Ann Hematol 85(8):489-501 (Aug)

18. Niscola P, Scaramucci L, Giovannini M, Anghel G, Romani C, Palombi F et al (2005) Palliative care in malignant hematology: an overview. Haema 8(2):297-315

19. Mercadante S (1997) Malignant bone pain: pathophysiology and treatment. Pain 69(1-2):1-18 (Jan)

20. Bruera E, Schoeller T, Wenk R et al (1995) A prospective multicenter assessment of Edmonton staging system for cancer pain. J Pain Symptom Manage 10(5):348-355 